\title{
Caracterización sensorial mediante perfil Flash de dos marcas de hot-dog de pollo comerciales y dos muestras formulados por ingeniería Kansei Tipo II
}

\section{Sensory characterization by Flash profile of two trademarks of chiken's hot-dog commercials and two samples formulated by Kansei engineering Type II}

\author{
Gustavo Gavino Puma Isuiza ${ }^{1 *}$ y Carlos Núñez Saavedra ${ }^{2}$
}

* Autor de correspondencia

\section{Resumen}

En la presente investigación se caracterizaron sensorialmente dos muestras de hot-dog de pollo comerciales y dos muestras obtenidas por Ingeniería Kansei tipo II, mediante el Perfil Flash. Los prototipos se elaboraron usando las siguientes operaciones: molienda, mezclado, cutterizado, embutido, escaldado, enfriado y escurrido. Los panelistas usaron entre 3 y 5 atributos, generando un total de 37 descriptores sensoriales, que contribuyeron a la diferenciación de las muestras dentro del espacio sensorial. El desempeño del panel se evaluó mediante el índice de consenso $(\mathrm{Rc}=0,592)$. Las dos primeras dimensiones del Análisis Procrustes Generalizado representaron el 92,85\% de la variabilidad de los datos. El Análisis de Varianza del Procrustes Generalizado (PANOVA) del Perfil Flash resultó significativo para la etapa de traslación $(p$-valor $=0,0001)$ y rotación $(p$-valor $=0,0004)$.

Palabras clave: Análisis Procrustes Generalizado (APG); caracterización sensorial; factor de escalamiento; hot-dog; Ingeniería kansei; Perfil Flash; PANOVA, Rc.

\begin{abstract}
In the present research two commercial hot-dog samples and two samples obtained by Ingeniería Kansei type II were characterized by the flash profile. The prototypes were made using the following operations: grinding, mixing, cutter, sausage, blanching, chilling and draining. The panelists used between 3 and 5 attributes, generating a total of 37 sensorial descriptors, which contributed to the differentiation of the samples within the sensory space. Panel performance was assessed using the consensus index $(\mathrm{Rc}=0.592)$. The first two dimensions of the Generalized Procrustes Analysis represented $92.85 \%$ of the variability of the data. The Generalized Procrustes Variance Analysis (PANOVA) of the Flash Profile was significant for the translation stage $(p$-value $=0.0001)$ and rotation $(p$-value $=0.0004)$.
\end{abstract}

Keywords: Flash Profile, Generalized Procrustes Analysis (GPA), hot-dog, kansei engineering, PANOVA, Rc, sensory characterization, scaling factor.

\section{Introducción}

Describir las características sensoriales de los productos ha sido una práctica común en la industria de alimentos desde hace mucho tiempo, ya sea para identificar el resultado del cambio de insumos o procesos, estimar la vida útil, asemejarse a un producto estándar, guiar la etapa de desarrollo de un nuevo producto o como medio de control de calidad. La caracterización sensorial es una de las herramientas más potentes, sofisticadas, y extensamente aplicada en la ciencia sensorial, ya que proporciona una representación de los aspectos cualitativos y cuantitativos de la percepción humana, que permite la medición de la reacción sensorial a los estímulos generados por un producto (Stone y Sidel 2004; Lawless y Heymann 2010; Moussaoui y Varela 2010; Varela y Ares 2014).
Los enfoques descriptivos convencionales son referencias indiscutibles de elaboración de perfiles sensoriales; sin embargo, se encuentran con limitaciones que retrasan o impiden las investigaciones. Algunas de estas limitaciones son intrínsecas al análisis descriptivo y se relacionan con la dificultad de medir la percepción, el tiempo de entrenamiento y los recursos necesarios para formar y mantener el panel descriptivo (Varela y Ares, 2014; Bruzzone, 2014).

El desarrollo de los métodos de Perfil de Libre Elección (PLE) y el Repertorio de Rejilla en los años 80 fue un punto de inflexión en la era sensorial, ya que permitió la utilización de consumidores y evaluadores no entrenados para la descripción sensorial. En este contexto, Sieffermann en el año 2000 ideó el Perfil Flash que combina los principios del PLE y la Evaluación 
Comparativa en Conjunto. La originalidad del Perfil Flash está en el hecho de que fue diseñado como un método de una sola medición, con énfasis en el posicionamiento sensorial relativo de los productos evaluados. Los datos generados por el Perfil Flash son procesados mediante el Análisis Procrustes Generalizado (Naes y Risvik, 1996; Dairou y Sieffermann, 2002; Valera y Ares, 2014; Delarue, 2015; Meullenet et al., 2007).

La Ingeniería Kansei (IK) tipo II es una técnica especialmente centrada en el usuario que se basa en trasladar y plasmar las imágenes mentales, percepciones, sensaciones y gustos del consumidor en los elementos del diseño que componen un producto, logrando incorporar los valores emocionales que atraen al consumidor, de forma cuantitativa (Nagamachi, 2011; Nagamachi y Mohd, 2011).

Por lo expuesto el objetivo de la investigación fue caracterizar mediante el Perfil Flash, dos hot-dog de pollo comerciales que se expenden en Lima Metropolitana y dos muestras de hot-dog de pollo obtenidas por Ingeniería Kansei Tipo II en la Facultad de Industrias Alimentarias de la UNALM.

\section{Materiales y métodos}

\section{Lugar de Ejecución}

La investigación se realizó en la Planta Piloto de Tecnología de Alimentos y Productos Agropecuarios y el Laboratorio de Evaluación Sensorial de la Facultad de Industrias Alimentarias de la Universidad Nacional Agraria La Molina (UNALM), Lima-Perú, desde agosto del 2015 a noviembre del 2016.

\section{Materia prima e insumos}

La materia prima principal fue pechuga de pollo (Gallus gallus); los insumos fueron: grasa dorsal de cerdo, hielo, sal (Ensal), polifosfato de sodio (Alitecno), pimienta, nuez moscada y comino esterilizados (GLM), color carmín natural (Frutaron), fundas artificiales (Alitecno), fundas comestibles (Quimsa) y sal de cura al 20\% (Alitecno). Los hot-dog de pollo comerciales fueron de las marcas: Otto Kunz y Braedt.

\section{Materiales y equipos \\ Materiales}

Cuchillo y olla $(20 \mathrm{~L})$ de acero inoxidable, termómetro (0 - $\left.100{ }^{\circ} \mathrm{C}\right)$, pipeta volumétrica $(1 \mathrm{~mL})$, beaker $(200 \mathrm{~mL})$, vasos de plástico (2 onzas), platos descartables (tamaño 3), marcadores indelebles, fichas de evaluación y lapiceros.

\section{Equipos}

Máquina picadora de carne (Mainca, PM-70), cocina industrial (a gas, sin modelo), balanza analítica (OHAUS, ARA 520), cutter (Hobart, 84145), embutidora Manual (sin marca, 10 ICTEFIAL) y computadora (Intel ${ }^{\circledR}$ Pentium $\AA$ Dual CPU, 2 GB RAM).

\section{Metodología experimenta}

\section{Obtención de hot-dog de pollo}

Para la obtención de los dos prototipos de hot-dog de pollo, se aplicó la Ingeniería Kansei tipo II, siguiendo la metodología propuesta por Marco-Almagro (2011) que se presenta en la Figura 1.

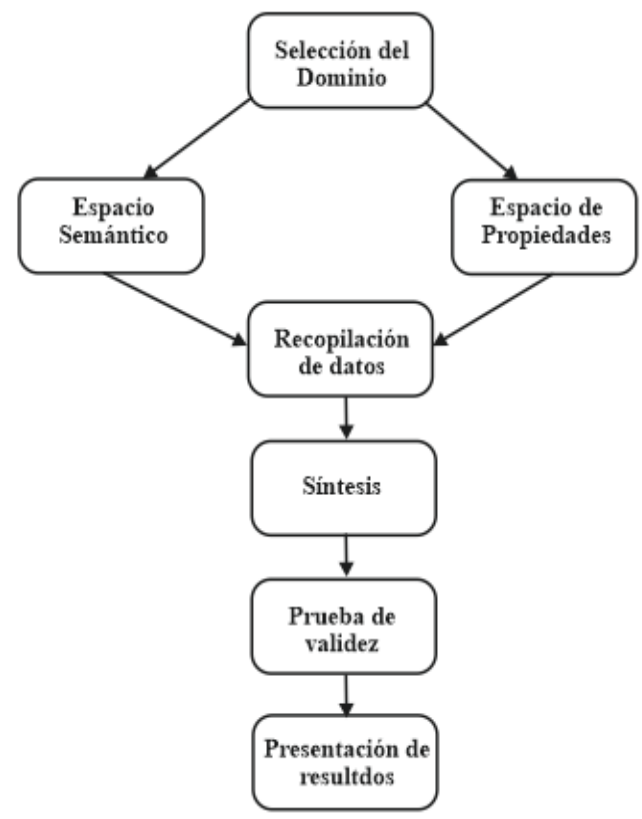

Figura 1. Modelo General de Ingeniería Kansei

Fuente: Marco-Almagro, 2011

\section{Elaboración de hot-dog de pollo}

Para la elaboración de los prototipos de hot-dog de pollo se siguió el procedimiento mostrado en la Figura 2, que se detalla a continuación (Elías et al., 2000):

Molienda, La carne de pollo y la grasa fueron molidas a través de un disco de $3 \mathrm{~mm}$ de diámetro.

Cutterizado, se agregó al cutter los insumos en el siguiente orden: carne de pollo, sal, sal de cura, eritorbato, polifosfato, la mitad del hielo, grasa, el resto del agua y los condimentos.

Embutido, Cuando la masa estuvo lista se embutió, en el equipo correspondiente, tratando de no introducir aire en las fundas.

Escaldado, Se escaldó en agua a $75-80^{\circ} \mathrm{C}$, hasta que la temperatura interna del producto alcanzó los $68-72^{\circ} \mathrm{C}$.

Enfriado, Se enfriaron los productos en agua helada (15 ${ }^{\circ} \mathrm{C}$ ) hasta que la temperatura en el punto más frío fue menor a $25^{\circ} \mathrm{C}$.

Escurrido, Se dejó escurrir el agua del hot-dog de pollo con ayuda de un colador.

\section{Perfil Flash}

Los panelistas reclutados fueron profesionales de la carrera de Ingeniería en Industrias Alimentarias de la FIAL o afines con experiencia en el desarrollo de perfiles de sabor, perfil 


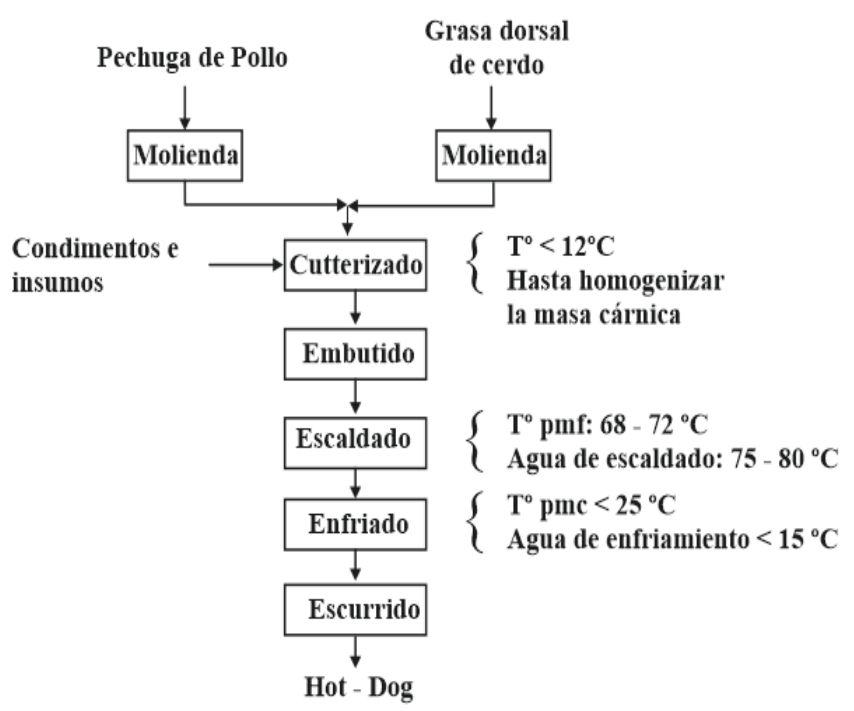

Figura 2. Flujo de operaciones para la obtención de Hot-dog a base de carne de pollo

de textura o Análisis Descriptivo Cuantitativo (QDA), por lo que fueron capaces de entender las instrucciones del líder de panel y generar atributos discriminantes y no hedónicos. Para llevar a cabo el Perfil Flash de las muestras de hotdog de pollo se siguieron los lineamientos establecidos por Varela y Ares (2014). Para ello, como primer paso, antes de iniciar la evaluación de las muestras se les brindó información sobre el fundamento de la metodología del Perfil Flash; además, se presentó un ejemplo práctico para el mejor entendimiento, teniendo en cuenta que este no tuvo influencia con el producto en estudio. También se les brindó información sobre las características texturales presentes en los alimentos.

En la etapa obtención de atributos se indicó a los panelistas que se centren en las diferencias entre los productos y que las diferencias detectadas las escriban en la ficha de evaluación. Esto implicó que los panelistas dieran prioridad directamente sobre las principales diferencias entre los productos. Se tuvo cuidado en que el panelista no se quede sin muestra al generar los atributos; $y$ en caso que fuese necesario, se les brindó más muestra. Se pidió a los panelistas que observen, manipulen y prueben las muestras con el fin de describir las características texturales que diferencian a los productos entre sí. Se les solicitó que utilizaran cualquier atributo no hedónico que consideraran apropiado para describir las muestras, siempre que sea suficientemente discriminativo para permitir una clasificación de las muestras (o una clasificación parcial).
En la etapa evaluación de las muestras se presentaron las cuatro muestras de hot-dog de pollo, en simultáneo y codificadas con letras, teniendo en cuenta que fueran homogéneas en cantidad y temperatura. Los panelistas ordenaron las muestras para cada atributo, con vinculaciones permitidas. El panelista pudo elegir anclajes que él encontró apropiados para evaluar los atributos sensoriales. Además, fueron libres de evaluar las muestras de hot-dog de pollo; tantas veces como consideraron necesario. Los panelistas realizaron la evaluación a su propio ritmo y fueron libres de tomarse un descanso en cualquier momento de la evaluación (Dairou y Sieffermann, 2002; Delarue y Sieffermann, 2004; Varela y Ares, 2014).

\section{Diseño experimental}

El diseño experimental para la caracterización de los prototipos de hot-dog de pollo se muestra en la Figura 3.

Análisis estadísticos. El Análisis Procrustes Generalizado se ejecutó con el software XL-STAT 2014 versión de prueba. Cada panelista generó una matriz $\mathrm{X}_{\mathrm{k}}$ de datos, la cual consistió en $\mathrm{N}$ filas con las puntuaciones de los descriptores generados en la evaluación sensorial. Esta matriz contuvo $\mathrm{X}_{\mathrm{ijk}}$ elementos, donde $\mathrm{i}$ es el índice sobre la cantidad de muestras de hot-dog evaluados; $j=1, \ldots, M_{k}$, el número de atributos generados por el $\mathrm{K}$ panelista, $\mathrm{y} \mathrm{k}=$ $1, \ldots, \mathrm{K}$, el número de los panelistas (Varela y Ares, 2014; Naes y Risvik, 1996; Mak et al., 2013).

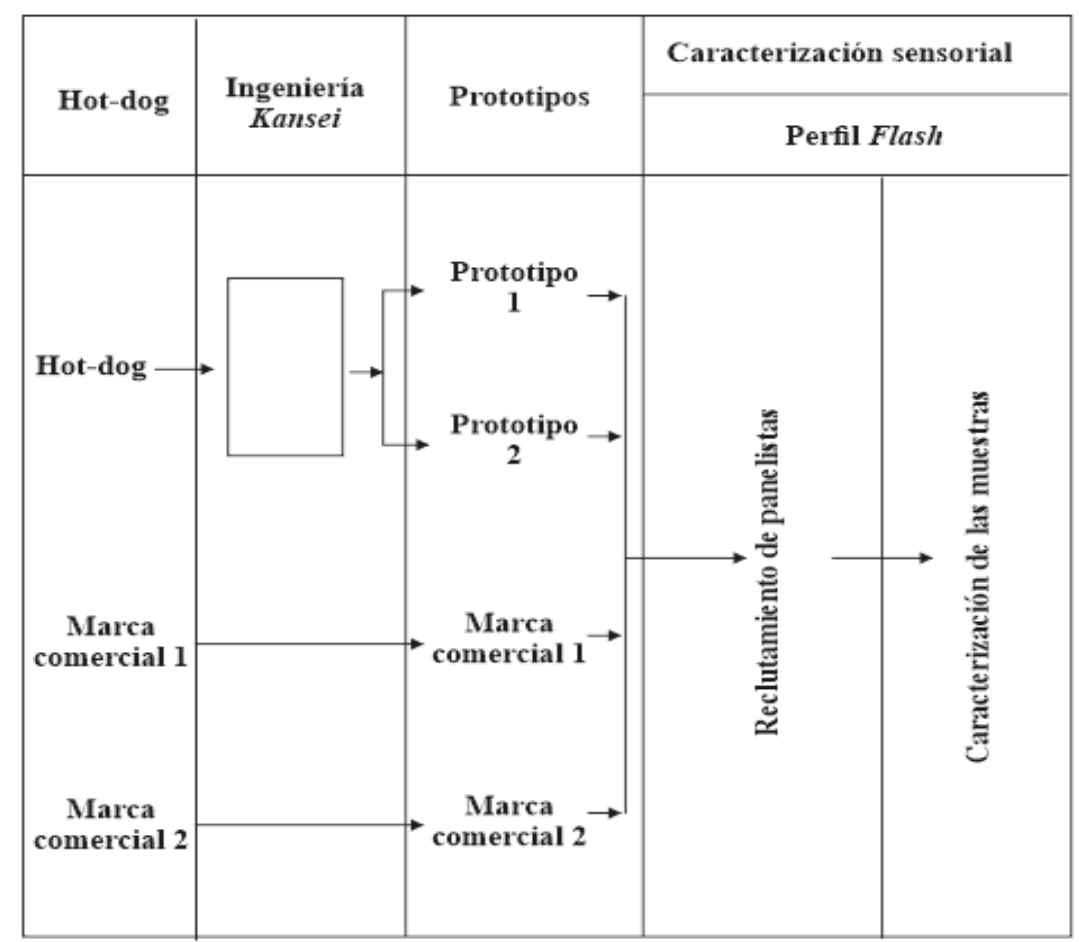

Figura 3. Diseño experimental para la caracterización sensorial de las muestras de hot-dog de pollo 


\section{Resultados y discusión}

Conformación del panel para la evaluación sensorial mediante el Perfil Flash

El panel para realizar el Perfil Flash estuvo conformado por nueve panelistas, de los cuales cuatro fueron estudiantes del Doctorado de Ciencia de los Alimentos, dos estudiantes de la Maestría de Tecnología de alimentos y tres estudiantes de la Facultad de Industrias Alimentarias que regularmente asistían al curso de Métodos Avanzados de Evaluación Sensorial, de la UNALM. Varela y Ares (2014) indican que cuando se realiza el Perfil Flash con panelistas con experiencia, el número mínimo de panelistas es de cuatro a cinco; ya que en esta metodología las medidas son complementarias a diferencia de los perfilados convencionales en donde las medidas son repetitivas.

Cada panelista generó entre 3 a 5 descriptores haciendo un total de 37 descriptores sensoriales. Esta cantidad de descriptores es inferior a los reportados en la literatura, debido a que en la presente investigación la evaluación sensorial estaba direccionada para solamente atributos texturales, a comparación de otros estudios en donde se evalúa el producto en cuanto a apariencia, olor, aroma, sabor y textura. Rason et al. (2006) realizaron el Perfil Flash de 12 salchichas secas tradicionales con 6 panelistas, cada uno generó entre nueve y 19 atributos haciendo un total de 83 descriptores, de los cuales los relacionados a la textura fueron entre uno y cuatro, haciendo un total de 15 descriptores. Ramírez-Rivera et al, (2016) realizaron el Perfil Flash de hamburguesas con cinco panelistas, cada uno generó entre nueve y 16 atributos, haciendo un total de 54 descriptores. Delaure y Sieffermann (2004) trabajaron con nueve y 10 panelistas para evaluar yogurt de fresa y queso fresco de albaricoque, respectivamente, generando entre cinco y nueve descriptores, haciendo un total de 38 y 29 descriptores, respectivamente.

Los tiempos de evaluación fueron aproximadamente de 25 y 45 minutos para la etapa de generación de descriptores y posicionamiento relativo, respectivamente, haciendo un total de 70 minutos, considerando que ningún panelista tomó descanso en la evaluación de los productos. Rason et al. (2006) en su investigación con 12 salchichas secas tradicionales reportaron que la duración total del Perfil Flash fue entre 105 a 120 minutos. Además, Varela y Ares (2014) mencionan que un Perfilado Flash puede variar entre 40 a 120 minutos de evaluación.

\section{Análisis de Varianza del Procrustes Generalizado (PANOVA)}

En la Tabla 1 se muestra el Análisis de Varianza del Procrustes Generalizado (PANOVA, por sus siglas en inglés), donde se resume la eficiencia de cada transformación del Análisis Procrustes Generalizado en términos de reducción de la variabilidad total (Silva et al., 2016; Tomic, 2013).
Tabla 1. Análisis de Varianza del Procrustes Generalizado (PANOVA)

\begin{tabular}{lccccc}
\hline \multicolumn{1}{c}{ Fuente } & $\begin{array}{c}\text { Grados } \\
\text { de } \\
\text { libertad }\end{array}$ & $\begin{array}{c}\text { Suma de } \\
\text { cuadrados }\end{array}$ & medios & F & p-valor \\
\hline $\begin{array}{l}\text { Residuos después } \\
\text { del escalamiento }\end{array}$ & 32 & 19,0446 & 0,5951 & & \\
$\begin{array}{l}\text { Escalamiento } \\
\begin{array}{l}\text { Residuos después } \\
\text { de la rotación }\end{array}\end{array}$ & 8 & 1,4274 & 0,1784 & 0,2998 & 0,9606 \\
$\begin{array}{l}\text { Rotación } \\
\begin{array}{l}\text { Residuos después } \\
\text { de la traslación }\end{array}\end{array}$ & 120 & 163,7778 & 1,3648 & & \\
$\begin{array}{l}\text { Traslación } \\
\text { Total corregido }\end{array}$ & 40 & 88,4720 & 0,5118 & & \\
\hline
\end{tabular}

La etapa de traslación ( $p$-valor $=0,0001$ ) obtuvo un impacto preponderante en la reducción de la variabilidad de las configuraciones, seguida de la etapa de rotación ( $p$-valor $=0,0004)$; sin embargo, la etapa de escalamiento $(p$-valor $=0,9606)$ no tuvo efecto significativo en la contribución de la reducción de la variabilidad de las configuraciones.

La Figura 4 presenta los residuos de cada panelista luego de la evaluación del Análisis Procrustes Generalizado (APG), donde el residuo fue mayor para el panelista 2 $(3,11)$, lo que indicó que dicho panelista estuvo más alejado del consenso. Similar comportamiento se observó en los panelistas $4(2,85), 1(2,63)$ y $7(2,23)$, seguidos de los panelistas 8, 9, 5 y 6 quienes obtuvieron 1,92, 1,91, 1,75 y 1,65, respectivamente. El panelista 3 fue quien obtuvo menos residuo $(0,96)$ lo que indicó que estuvo más cerca al consenso del panel.

En la Figura 5 se muestra los factores de escalamiento isotrópico del APG. Los panelistas 7 y 9 obtuvieron un factor de escalamiento mayor a 1 (1,19 y 1,20 , respectivamente) lo que indica que sus configuraciones han sido estiradas para llegar al consenso, debido a que calificaron algunos atributos con la parte inferior de la escala; además, son los que generaron menor cantidad de atributos texturales.

Los panelistas 1, 2, 3, 4 y 5 presentaron un factor de escalamiento menor a $1(0,98,0,97,0,97,0,89$ y 0,90 ,

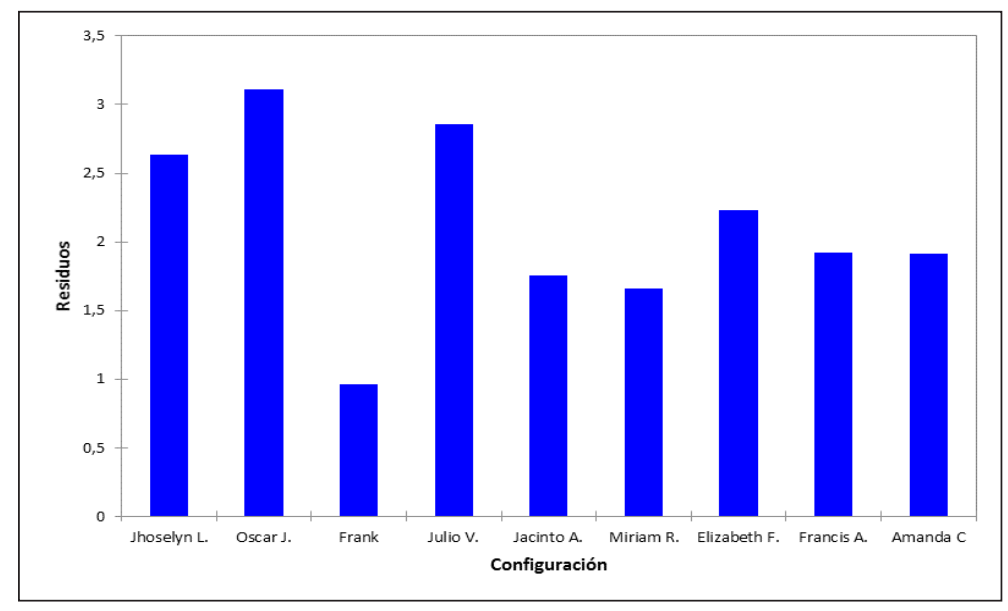

Figura 4. Residuos de los panelistas luego de la evaluación APG 
respectivamente), lo que indica que; sus configuraciones han sido reducidas para llegar al consenso. Los panelistas 6 y 7 obtuvieron un factor de escalamiento cercano a $1(1,00$ y 1,03 , respectivamente) por lo que sus configuraciones no han sufrido mayor modificación en la etapa de escalamiento del APG. Los resultados son similares a los datos reportados por Silva et al., (2016) quienes obtuvieron factores que oscilan entre $0,77 \mathrm{y}$ 1,25, aproximadamente.

En la Figura 6 se observan los residuos para cada muestra después de las transformaciones del APG. La muestra $\mathrm{C}$ obtuvo el menor residuo $(3,40)$, lo cual indicó que no hubo gran diferencia entre las calificaciones de los panelistas para esta muestra. Por otra parte, el mayor residuo se obtuvo para el hot-dog Otto Kunz $(7,13)$ es decir, se presentó un menor consenso entre los panelistas respecto a esta muestra.

\section{Determinación del consenso de los panelistas mediante el índice Rc}

Para la determinación del consenso de los panelistas se utilizó el índice de consenso $R c$ (Figura 7). El $R c$ para los panelistas fue de $0,592(59,2 \%)$; este valor indicó una correlación positiva, es decir, se generó un adecuado consenso en el desempeño de los panelistas. Dicho valor fue superior a los reportado por Hernández et al. (2010) en la evaluación de quesos con jueces no entrenados $(R c=55,3 \%)$ y Silva et al. (2016) en la evaluación con consumidores de una salsa a base de hongos (Rc 56,0\%); sin embargo, fue inferior a lo reportado por Ramírez et al. (2011) en la caracterización sensorial de frituras tipo chip $(R c=78,1 \%)$ y Wu et al. (2002) en la descripción sensorial de yogurt mediante el Perfil de Libre Elección $(R c=77,7 \%)$.

En la Figura 8se observa la ubicación de los cuatro productos y del consenso, evaluados por los nueve panelistas, cada uno en diferentes cuadrantes respecto a las dimensiones F1 y F2 (92,85\%). En la Figura 9 se observa que la primera dimensión estuvo relacionada principalmente con los descriptores: dureza, arenosidad, pegajosidad, granulosidad y grasoso. La segunda dimensión fue explicada mejor por el término: masticabilidad. Según Kobayashi y Benassi (2012) solo los descriptores con coeficientes de correlación igual o superior a 0,60 (valor absoluto) y a la vez citados por varios panelistas, deben ser utilizados para visualizar las relaciones entre las muestras y los atributos.

El resultado del análisis del Perfil Flash representado en dos dimensiones $(\mathrm{F} 1=69,82$ y F2 $=23,03)$ explicó el APG

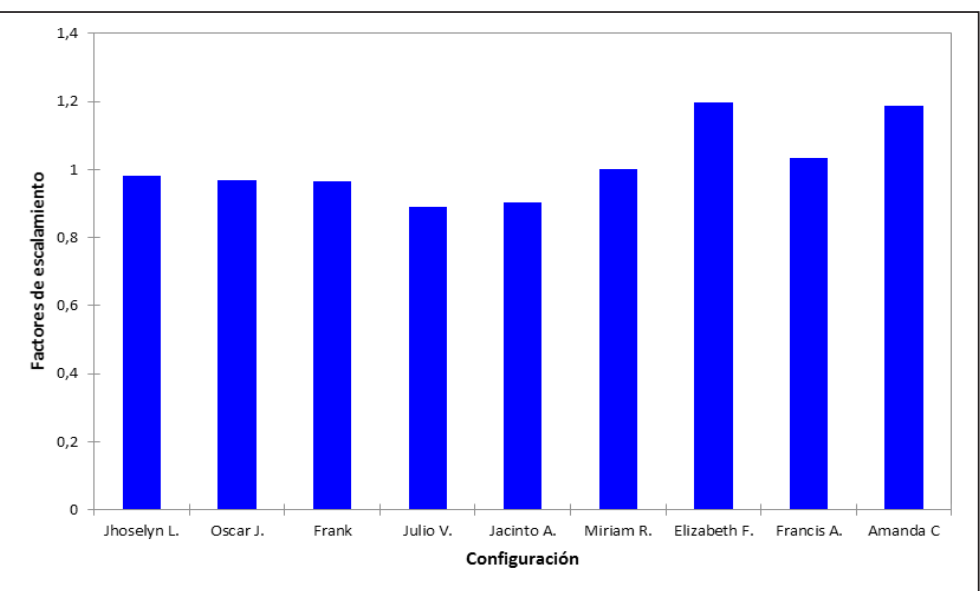

Figura 5. Factores de escalamiento isotrópico del APG de cada panelista.

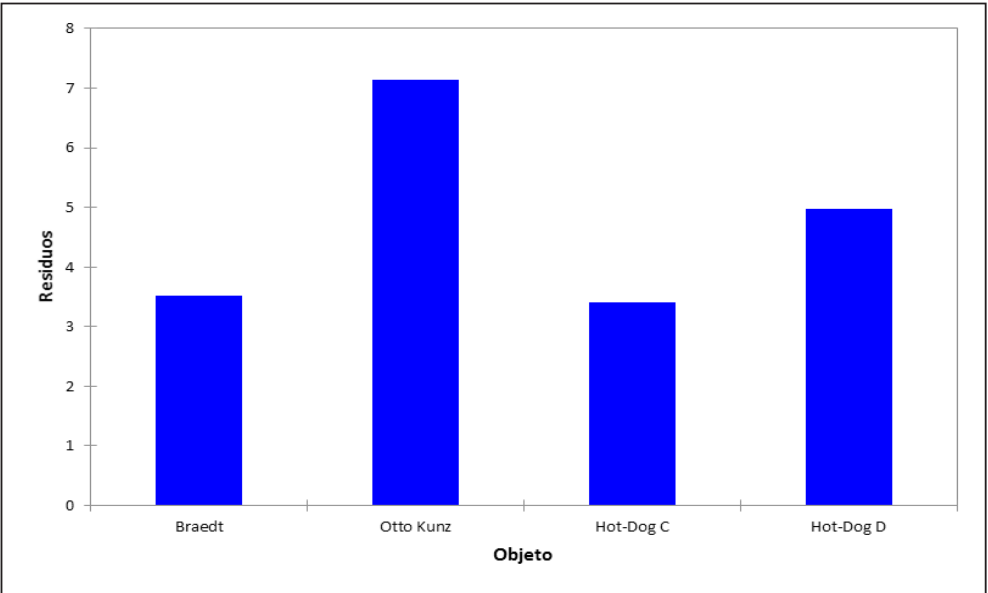

Figura 6. Residuo por muestra después de las transformaciones del

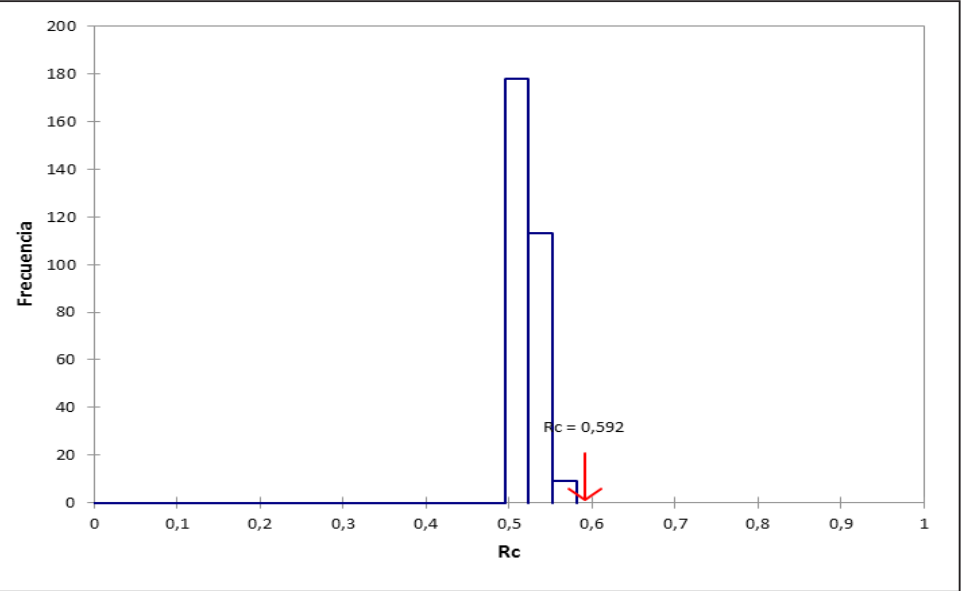

Figura 7. Índice de consenso (Rc) del panel de Perfil Flash

$92,85 \%$ de la variabilidad de los datos (Figura 9). Este valor fue superior a lo obtenido por Rason et al. (2006) en la caracterización sensorial de salchichas secas tradicionales (78,0\%), Ramírez et al. (2010) en la caracterización sensorial de hamburguesas de pescado $(83,23 \%)$, Ramírez et al. (2009) en la caracterización sensorial del camarón ahumado (82,39\%), Gamboa et al. (2012) en 


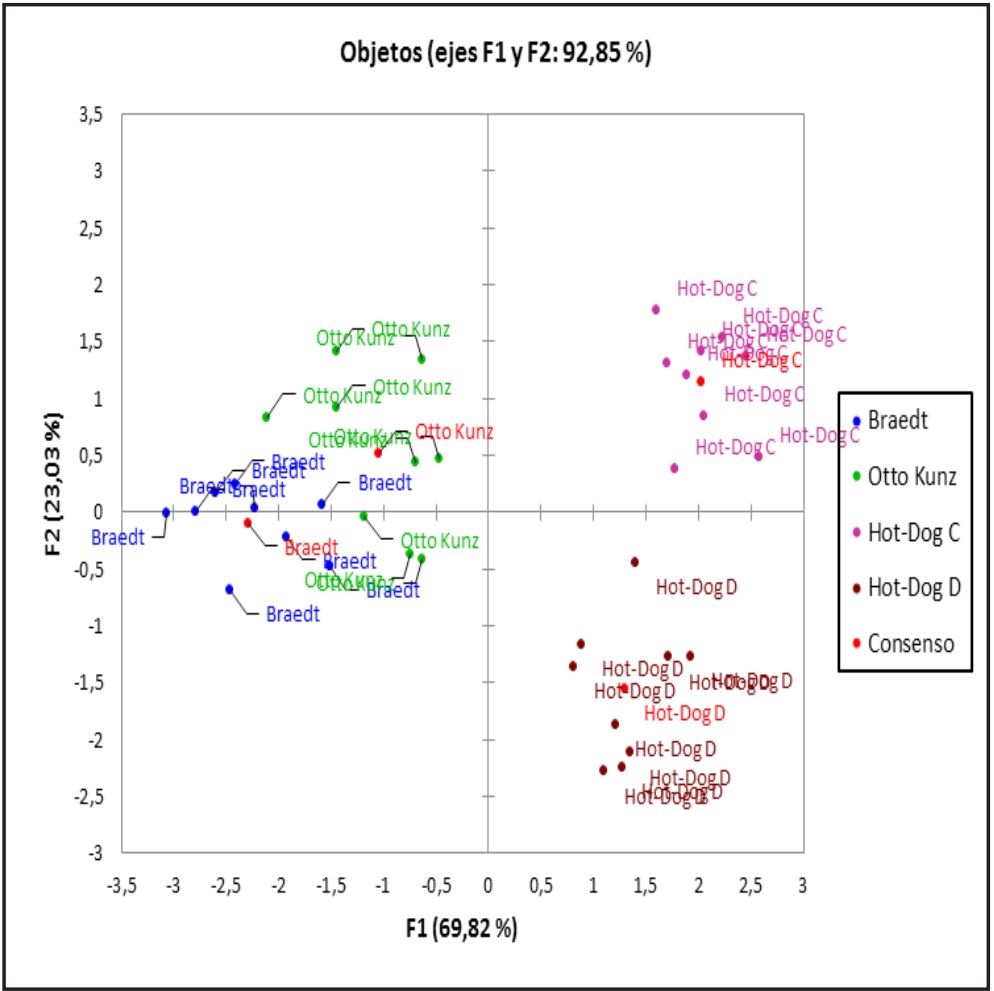

Figura 8. Espacio sensorial y de consenso de las cuatro muestras de hot-dog

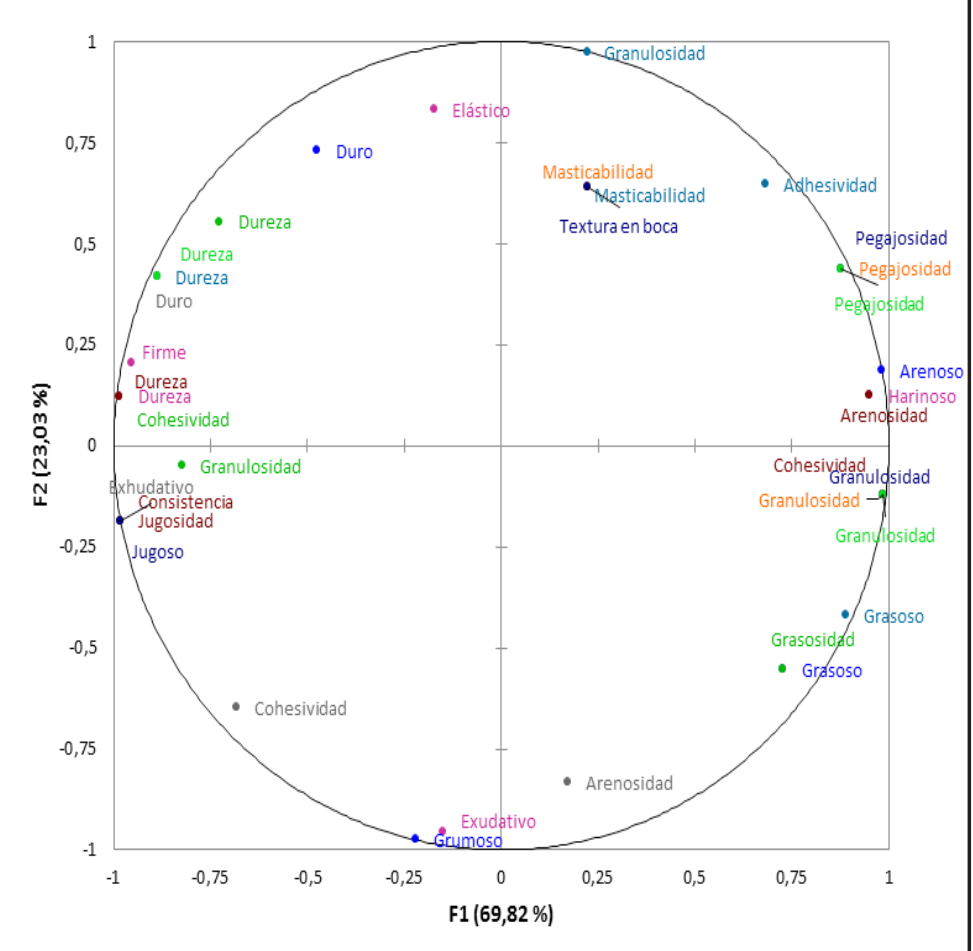

Figura 9. Espacio sensorial de los atributos del panel de Perfil Flash
Relacionando la Figura 8 y Figura 9, la muestra $\mathrm{C}$, fue caracterizada con los descriptores: pegajosidad, arenosidad (Componente F1 positivo), granulosidad y masticabilidad (Componente F2 positivo). La muestra D fue caracterizada con los descriptores: granuloso y grasoso (Componente F1 positivo). La marca Braedt fue caracterizada por los descriptores: jugosidad (Componente F1 negativo) y cohesividad (Componente F2 negativo). La marca Otto Kunz fue caracterizada por el descriptor: dureza (componente F1 negativo).

\section{Conclusiones}

La aplicación del Perfil Flash en la caracterización de hot-dog de carne de pollo permitió un rápido acceso al posicionamiento sensorial de los productos y atributos que contribuyeron a diferenciar las muestras, ya que esta metodología permite eliminar las sesiones de entrenamiento del panel, reduciendo tiempo y costos. Los descriptores generados mediante el Perfil Flash para las muestras de hot-dog de pollo fueron: dureza, arenosidad, pegajosidad, granulosidad, grasoso y masticabilidad.

\section{Literatura citada}

Bruzzone, F. 2014. Aplicación de Metodologías de Caracterización Sensorial Con Consumidores en el Desarrollo de Postres Lácteos Funcionales. Tesis Para Optar El Título de Magister en Ciencias y Tecnología de Alimentos. Universidad de la República. Facultad de Química - Facultad de Ingeniería. $113 \mathrm{P}$.

Dairou, V. and Sieffermann, J. 2002. A Comparison of 14 Jams Characterized by Conventional Profile and a Quick Original Method, The Flash Profile. Journal of Food Science 67(2):826-834.

Delarue, J. and Sieffermann, J. 2004. Sensory Mapping Using Flash Profile. Comparison With a Conventional Descriptive Method for The Evaluation of the Flavour of Fruit Dairy Products. Food Quality and Preference 15 (2004) 383-392.

Delarue, J. 2015. Flash Profile, its Evolution and uses In Sensory and Consumer Science.

la determinación de atributos sensoriales de queso tipo Manchego durante la maduración $(72,69 \%)$, Dairou y Sieffermann (2002) en la comparación de 14 mermeladas caracterizadas por QDA ${ }^{\circledR}$ y Perfil Flash $(69,0 \%)$ y Silva et al. (2016) en la caracterización de salsa a base de hongo $(82,60 \%)$.
Applications In New Product Development and Consumer Research. A volume In Woodhead Publishing Series In Food Science, Technology and Nutrition. 121-151 P.

Elías, C.; Chirinos, R. y Salvá, B. 2000. Elaboración de 
Embutidos. Lima, Perú. 91p.

Gamboa, J.; Guadalupe, D. y Ramírez, E. 2012. Calidad Fisicoquímica y Sensorial de Queso Tipo Manchego Durante la Maduración. Revista Cientifica Udo Agrícola 12(4):929-938.

Hernández, M.; López, J.; Gómez, T.; Santiago, R.; Ramón, L.; Delgado, F.; Shain, A.; Huante, Y. y Ramírez, E. 2010. Comparación de la Descripción Sensorial del Queso Fresco "Cuajada" Mediante el Análisis Descriptivo Cuantitativo y el Perfil Flash. Ciencia y Mar 2010, Xiv 42: 3-12.

Kobayashi, M. y Benassi, M. 2012. Caracterização Sensorial de Cafés Solúveis Comerciais Por Perfil Flash. Semina: Ciências Agrárias.33(2):3081-3092.

Lawless, H. y Heymann, H. 2010. Sensory Evaluation of Food. Principles and Practices. Segunda Edición. Editorial. Springer. New York, Usa. 596P.

Mak, A.; Lumbers, M.; Eves, A. and Chang, R. 2013. An Application of The Repertory Grid Method and Generalised Procrustes Analysis To Investigate The Motivational Factors of Tourist Food Consumption. International Journal of Hospitality Management 35. 327-338 P.

Marco-Almagro, L. 2011. Statistical Methods In Kansei Engineering Studies (Tesis Para Optar el grado de Doctor). Universidad Politécnica de Catalunya. Barcelona, España.

Meullenet, J.; Xiong, R. y Findlay, C. 2007. Multivariate and Probabilistic Analyses of Sensory Science Problems. Ift Press Ed. Advisory Board. 248 P.

Moussaoui, K. and Varela, P. 2010. Exploring Onsumer Product Profiling Techniques and Their Linkage To A Quantitative Descriptive Analysis. Food Quality and Preference 21. 1088-1099.

Nagamachi, M. 2011. Kansei/Affective Engineering. CRC Press. Taylor \& Francis Group. 309 P.

Nagamachi, M. and Mohd, A. 2011. Innovations of Kansei Engineering. Crc Press. Taylor \& Francis Group. 151 P.

Naes, T. and Risvik, E. 1996. Multivariate Analysis of Data In Sensory Science. Elsevier Science B.V. 16. 348.

Ramírez, E.; Ramón, L.; Huante, Y.; Shaín, A.; Bravo, H. y Martínez, C. 2009. Caracterización Sensorial del Camarón Ahumado (Litopenaeus Vannamei) Mediante la Técnica Perfil Flash. Ciencia y Mar. Xiii (38):27-34 P.

Ramírez, E.; Ramón, L.; Camacho, M.; Reyes, V.; Rodríguez, M. y Shaín, A. 2010. Correlación Entre el Perfil Descriptivo Cuantitativo y Perfil Flash de Hamburguesas de Pescado de Barrilete Negro (Euthynnus Lineatus). Nacameh. 4(2):55-68.

Ramírez, E.; Paz, E. y Nogueira, H. 2011. Caracterización Sensorial y Análisis de las Preferencias de los Consumidores de Frituras Tipo Chips de Malanga (Colocasia Esculenta). Revista Venezolana de Ciencia y Tecnología de Alimentos 2 (2): 277-292.

Ramírez-Rivera, E. D. J.; Juárez-Barrientos, J. M.; Rodríguez-Miranda, J.; Ramírez-García, S. A.; VillaRuano, N. \& Ramón-Canul, L. G. 2016. Comparación de mapas de preferencia mediante el análisis descriptivo cuantitativo y perfil flash en hamburguesas.Ecosistemas y recursos agropecuarios 3(7), 103-112.

Rason, J.; Léger, L. Dufour, E. and Lebecque, A. 2006. Relations Between The Know-How of Small-Scale Facilities and The Sensory Diversity of Traditional Dry Sausages From The Massif Central In France. Eur Food Res Technol. 222: 580-589 P.

Silva, R.; Huamán, M.; Hurtado De Mendoza, K. y Bravo, N.; Silva, A. 2016. Caracterización Sensorial de Salsa a Base de Hongos (Pleurotus Ostreatus) Mediante La Técnica Perfil Flash. Revista Venezolana de Ciencia y Tecnología de Alimentos 7 (1): 001-016 P.

Stone, H. and Sidel, J. 2004. Sensory Evaluation Practices. Tercera Edición. London, U.K.: Elsevier Academic Press. 377 P.

Tomic, O. 2013. Differences Between Generalised Procrustes Analysis and Multiple Factor Analysis In The Case Of Projective Mapping. (Tesis Para Optar el grado de Magíster ). Department of Chemistry, Biotechnology And Food Science Norwegian University of Life Sciences.

Varela, P. and Ares, G. 2014. Novel Techniques In Sensory Characterization and Consumer Profiling. Crc Press. Taylos and Francis Group. London, New York. 389 P.

Wu, W.; Guo, Q.; De Jong, S. and Massart, D.L. 2002. Randomisation Test For The Number O Dimensions Of The Group Average Space In Generalised Procrustes Analysis. Food Quality and Preference 13(3):191-200 . 